\section{Chromosomes and malignancy}

ABNORMAL chromosomes are a regular feature of tumour cells. Non-transformed cells rarely show chromosomal abnormality unless obtained from developmentally defective individuals, many of whom show an increased predisposition to cancer. Altered chromosomes therefore either predispose oncogenic change or accompany it. Most cancer-producing agents cause chromosome damage, which may therefore be an important aspect of their potency for tumour induction. But the majority of such damaged cells are inviable, a few are transformed and only a proportion of these may have malignant properties. These wide ranging manifestations of the transformed state have attracted the attention of many workers seeking some explanation in terms of the accompanying chromosomal alterations.

Sachs and his group (Hitotsumachi, Rabinowitz and Sachs, Nature, 231, 511; 1971) have shown that the in vitro propagation of polyoma transformed cells may be accompanied by loss of malignant characteristics and the appearance of new groups of chromosomes which are suggested to carry suppressors of malignancy; the balance of these chromosomes in the nucleus is supposed to be an important feature of its control. Harris and his coworkers (Klein, Bregula, Weiner and Harris, J. Cell Sci., 8, 659; 1971; Weiner, Klein and Harris, J. Cell Sci., 12, 253; 1973) have fused together cells with and without malignant properties. The result of such an experiment is usually suppression of malignancy, followed by its restoration after the loss of chromosomes from the normal, non-malignant, parent. Fusing together two malignant cells however usually resulted in a malignant cell without the necessity for chromosomal loss. In certain cases the latter experiment resulted in loss of malignancy (Harris, Proc. R. Soc., B179, 1; 1971), all of which leads Harris to stress the role of individual chromosomes which carry malignancy suppressors as the important feature of its control. Both groups invoke chromosomally based factors as the essential elements, although in neither case are particular chromosomes designated as the carriers.

In this issue of Nature however (page 610) Codish and Paul report the reversible appearance of a specific chromosome which suppresses malignancy in a chemically induced tumour. When present in two copies, both malignancy and antigenicity are suppressed, but both are restored when only a single copy is present. The extra chromosome is thought to originate from the fusion of part of a normal number 19 mouse chromosome with an unaltered number 7. Since the normal elements of this marker chromosome are also present in the cells, it seems possible that the suppression involves a position effect following fusion of the two normal chromosome segments, although this is not suggested by the authors. There seemed to be no other consistent correlation with suppression of malignancy

A somewhat similar mechanism, resulting in the acquisition of malignancy rather than its suppression, is suggested in the case of the now classical examples of human tumours: chronic myeloid leukaemia in which $90 \%$ of cells exhibit a loss of most of the long arm from chromosome 22 (Caspersson, Gahrton and Lindsten, Exp. Cell. Res., 63, 238; 1970) and the acquisition of a similar fragment by the number 9 chromosome (Rowley, Nature, 243, 290; 1973); and meningiomas in which there is also loss of part of the number 22 chromosome (Zankl and Zang, Humangenetik, 14, 167; 1972). Other cases of specific abnormalities include the number 14 chromosome with an additional terminal fluorescent band in tumours of some patients with Burkitt's lymphoma (Manolov and Manolova, Nature, 237, 33; 1972), and the number 13 interstitial deletion in retinoblastoma (Wilson, Towner and Fujimoto,
Am. J. hum. Genet., 2, 212; 1973). Not surprisingly, some tumours seem not to exhibit specific alterations in chromosomes, but show random changes (Popescu et al. Int. J. Cancer, 14, 461; 1974) and others exhibit variability in malignancy correlated with the fluctuation in numbers of heterochromatic minute chromosomes (Shepard et al., Cytogenet. Cell Genet., 13, 279; 1974). The interest of the material described by Codish and Paul lies in the apparently clear-cut nature of the phenomenon and one hopes that they will take advantage of the cell fusion approach to analyse it in more detail.

The tendency of certain tumours to exhibit predictable alterations in certain chromosomes, whether connected with increased malignancy or its suppression, presumably reflects a number of related factors such as the probability of the alteration occurring, the viability of the altered state and the predilection of certain carcinogens for causing damage at selected sites. It may be that certain chromosomes (for example those involved in nucleolus organisation) come more frequently into contact than others thus increasing the probability of interchange.

It seems quite certain however that particular groups of chromosomes are more frequently involved in formation of the marker chromosomes typical of a cell line than are others following virus transformation by adenovirus, SV40 and herpesvirus (Gallimore, cited in McDougall, Prog. med. Virol., in the press). Chemical transformation of rat cells also results in the preferential involvement of three autosomes in marker formation (Olini and DiPaolo, J. natn. Cancer Inst., 52, 627; 1974) and two of these are regularly involved in the genesis of markers in adenovirus transformed cells (McDougall, Prog. Med. Virol., in the press). An important future task will be to establish the mechanism of this effect. For this purpose virus-transformed cells seem ideal since they permit the use of specific probes which can detect integrated viral genomes, their transcriptional and translational products at the cell level. A beginning to such work has been made by the localisation of the control of SV40 T antigen to human chromosome 7 (Croce, Girardi and Koprowski, Proc. natn. Acad. Sci, U.S.A., 70, 3617; 1973). Progress should be spectacular in view of the recent advances in analysis of the transforming genes of adenoviruses (see Rogers, Nature, 251, 668; 1974).

K. W. JONES

\section{Arrival of the age of Rama}

Ir is now generally accepted that the survival of the human race is threatened by twentieth century technology. Mankind must live with the uncertain hope that future innovation will not create more dangers than it removes, and occurs rapidly enough to forestall the collapse of organised industrial society.

Speculative science fiction enthusiasts have long dreamed of preserving humanity from eanthbound destruction by colonising other planets. The stunning successes of near space exploration over the last decade have moved the discussion of extraterrestrial migration out of the domain of pure fantasy into the 'light relief' area of established science. But most of the colourful accounts published assume a depressingly high level of technology, in many cases decades or even centuries ahead of our present development. It is not now at all clear that technology will ever be able to reach the level at which exotic extraterrestrial solutions, such as the colonisation of Mars, will provide for the continuation of the human species. The global crisis has apparently arrived a fiew decades too soon.

If there is little ground for confidence in further gigantic technological leaps in the foreseable future, it is heartening 